\title{
An intranasal influenza vaccine for the prevention of influenza in healthy children was cost effective
}

Luce BR, Zangwill KM, Palmer CS, et al. Cost-effectiveness analysis of an intranasal influenza vaccine for the prevention of influenza in healthy children. Pediatrics 2001 Aug;108:e24.

\section{QUESTION: In healthy children, is an intranasal influenza vaccine for the prevention of influenza cost effective?}

\section{Design}

Randomised \{allocation concealed $*\}$, blinded \{patients, healthcare providers, data collectors, judicial assessors of outcomes, data analysts, data safety and monitoring committee, and manuscript writers $\} \uparrow, *$ placebo controlled trial with 2 years of follow up.

\section{Setting}

University medical centres in 7 cities in the USA.

\section{Patients}

2960 healthy children who were 15 to 71 months of age (mean age $42 \mathrm{mo}, 52 \%$ girls). Follow up was $>97 \%$.

\section{Intervention}

Children were allocated to receive 1 or 2 doses of a live, attenuated, trivalent, intranasal influenza vaccine $(n=1987)$ or placebo $(n=973)$ for the $1996-1997$ and 1997-1998 seasons.

\section{Main cost and outcome measures}

Cost per febrile influenza-like illness (ILI) day avoided. A break-even analysis was also done to calculate the vaccine plus administration cost below which its use would be cost saving. Per child cost-effectiveness analyses were done separately for an individual based and group based vaccination delivery scenario from the societal and third-party payer perspectives. Costs were assessed in US dollars and were discounted at a rate of $3 \%$ in year 2 .

\section{Main results}

Over 2 years, children who received the vaccination had a mean of 1.2 fewer febrile ILI days/child than those who were unvaccinated. In an individual based vaccination scenario, the mean per child cost effectiveness was $\$ 29.67 /$ febrile ILI day avoided, assuming a vaccine plus administration cost of $\$ 20$. At vaccination plus administration costs of $\$ 10$ to $\$ 40 /$ dose, cost effectiveness ranged from $\$ 9.98$ to $\$ 69.03$, respectively, per febrile ILI day avoided. Break-even analyses showed that the vaccine plus administration cost below which its use would be cost saving was $\$ 28$ for a group based vaccination scenario and $\$ 4.93$ for an individual based vaccination scenario.

\section{Conclusion}

In healthy children, an intranasal influenza vaccine for the prevention of influenza may be cost effective.

*See glossary.

$\dagger$ Information provided by author.

\section{COMMENTARY}

In the USA, professional medical societies and the Advisory Committee on Immunization Practices are considering potential recommendations for routine influenza vaccine use in children. Discussions focus primarily on the effect of vaccination on serious disease in young infants. Costeffectiveness data may affect vaccination recommendations for older children.

The study by Luce $e t$ al using the intranasal influenza vaccine provides point estimates for cost effectiveness using 2 vaccination scenarios from various perspectives. Although the authors suggest that influenza vaccination costs would be reduced by group vaccination (eg, at day care) or administering a single dose in the first vaccination year, the former will probably not be acceptable to most paediatricians and the latter will probably be inconsistent with the schedule licensed by the US Food and Drug Administration. A strategy to reduce costs consistent with licensed indications would be to use a cheaper vaccine, whether it be the currently available injection or the intranasal preparation. Head to head comparisons of the 2 types of vaccines among healthy children and adults show no difference in efficacy. ${ }^{12}$

How much confidence can one place in cost-effectiveness estimates from a study done over 2 influenza seasons? Isolation of influenza viruses from patients in the placebo group was $44 \%$ higher in the first than in the second year, reflecting substantial variation in cost effectiveness by season. Cost effectiveness also varies with the match between the circulating virus and the vaccine strains. The effect of paediatric vaccination on disease transmission and infection in adults may result in substantial disease burden and cost reductions, but the relation between age-specific coverage and effectiveness is unknown.

Current recommendations include paediatric influenza vaccination issues that may affect the extent of intranasal vaccine cost, provider reimbursement, the feasibility of widespread seasonal immunisation in paediatrics, and parental acceptance of a vaccine that will reduce but not eliminate upper respiratory illness. Vaccine cost, a major factor in the results of this cost-effectiveness analysis, will probably be a key factor in answering these questions.

Ben Schwartz, MD Centers for Disease Control and Prevention Atlanta, Georgia, USA

1 Edwards KM, Dupont WD, Westrich MK, et al. A randomized controlled trial of cold-adapted and inactivated vaccines for the prevention of influenza A disease. J Infect Dis 1994;169:68-76.

2 Belshe RB, Gruber WC, Mendelman PM, et al. Efficacy of vaccination with live, attenuated, cold-adapted, trivalent, intranasal influenza virus vaccine against a variant (A) Sydney) not contained in the vaccine. $J$ Pediatr 2000;136:168-75. 